ARTICLE

\title{
Higher intake of whole grains and dietary fiber are associated with lower risk of liver cancer and chronic liver disease mortality
}

\author{
Xing Liu ${ }^{1,2}$, Wanshui Yang (10 ${ }^{3,4}$, Jessica L. Petrick (1) ${ }^{5}$, Linda M. Liao ${ }^{6}$, Weibing Wang ${ }^{2}, \mathrm{Na} \mathrm{He}^{2}$, \\ Peter T. Campbell ${ }^{7}$, Zuo-Feng Zhang (1) ${ }^{8,9}$, Edward Giovannucci, ${ }^{1,3,10}$, Katherine A. McGlynn ${ }^{6}$ \& \\ Xuehong Zhang (1,3凶
}

The relationship between dietary factors and liver disease remains poorly understood. This study evaluated the associations of whole grain and dietary fiber intake with liver cancer risk and chronic liver disease mortality. The National Institutes of Health-American Association of Retired Persons Diet and Health Study cohort recruited 485, 717 retired U.S. participants in 1995-1996. Follow-up through 2011 identified 940 incident liver cancer cases and 993 deaths from chronic liver disease. Compared with the lowest, the highest quintile of whole grain intake was associated with lower liver cancer risk (Hazard ratio $[H R]_{Q 5}$ vs. Q1 $=0.78,95 \%$ confidence interval $[\mathrm{Cl}]$ : 0.63-0.96) and chronic liver disease mortality $\left(\mathrm{HR}_{\mathrm{Q} 5}\right.$ vs. $\mathrm{Q}_{1}=0.44$, 95\% Cl: 0.35-0.55) in multivariable Cox models. Dietary fiber was also associated with lower liver cancer risk ( $\mathrm{HR}_{\mathrm{Q} 5}$ vs. Q1 $\left.=0.69,95 \% \mathrm{Cl}: 0.53-0.90\right)$ and chronic liver disease mortality $\left(\mathrm{HR}_{\mathrm{Q} 5}\right.$ vs. $\left.\mathrm{Q} 1=0.37,95 \% \mathrm{Cl}: 0.29-0.48\right)$. Fiber from vegetables, beans and grains showed potential protective effect. Here, we show that higher intake of whole grain and dietary fiber are associated with lower risk of liver cancer and liver disease mortality.

\footnotetext{
${ }^{1}$ Department of Nutrition, T.H. Chan School of Public Health, Harvard University, Boston, MA, USA. ${ }^{2}$ Department of Epidemiology, School of Public Health, Fudan University, Shanghai, P.R. China. ${ }^{3}$ Channing Division of Network Medicine, Department of Medicine, Brigham and Women's Hospital and Harvard Medical School, Boston, MA, USA. ${ }^{4}$ Department of Nutrition, School of Public Health, Anhui Medical University, Hefei, Anhui, P.R. China. ${ }^{5}$ Slone Epidemiology Center, Boston University, Boston, MA, USA. ${ }^{6}$ Division of Cancer Epidemiology and Genetics, National Cancer Institute, Bethesda, MD, USA. ${ }^{7}$ Behavioral and Epidemiology Research Group, American Cancer Society, Atlanta, GA, USA. ${ }^{8}$ Department of Epidemiology, Fielding School of Public Health, University of California, Los Angeles, CA, USA. ${ }^{9}$ Jonsson Comprehensive Cancer Center, UCLA, Los Angeles, CA, USA. ${ }^{10}$ Department of Epidemiology, T.H. Chan School of Public Health, Harvard University, Boston, MA, USA. ${ }^{凶}$ email: xuehong.zhang@channing.harvard.edu
} 
P rimary liver cancer is the sixth most common cancer and the third most common cause of cancer-related mortality worldwide $^{1}$, and its incidence and mortality have been increasing in the United States ${ }^{2}$. The five-year survival rate of liver cancer has only improved from $11.7 \%$ to $21.3 \%$ during $2000-2011^{3}$, suggesting the importance of primary prevention for this fatal disease. Hepatocellular carcinoma (HCC) and intrahepatic cholangiocarcinoma (ICC) are the two most common histologic types of primary liver cancer, accounting for $75-80 \%$ and $10-15 \%$ of cases, respectively 4,5 . Major known risk factors for HCC include chronic hepatitis B virus (HBV) infection, chronic hepatitis $\mathrm{C}$ virus (HCV) infection, aflatoxin, heavy alcohol consumption, tobacco smoking, obesity, nonalcoholic fatty liver disease (NAFLD), and type 2 diabetes mellitus (T2DM) ${ }^{6,7}$. These factors are also associated, although not as strongly, with ICC. Certain biliary tract conditions, including choledochal cyst, choledocholithiasis, and cholelithiasis, are strongly associated with ICC $^{8}$. Chronic liver diseases (CLD), including cirrhosis, fibrosis, alcoholic liver disease, and chronic hepatitis, are important precursors of HCC. Additionally, CLD may lead to death itself and is also a significant public health concern ${ }^{9}$. Non-HBV/non-HCVrelated HCC is common in the U.S., with the etiology poorly understood $^{6}$. More HCC and CLD are probably attributable to obesity, T2DM and NAFLD in this population ${ }^{6}$, which are closely associated with behaviors including dietary patterns and composites. Thus, identifying dietary factors that could reduce the risk of liver cancer and CLD mortality is of interest. However, to date, such research is limited ${ }^{10,11}$.

Whole grains contain the endosperm, as do refined grains, but also bran and germ. Thus, whole grains serve as valuable food sources of dietary fiber, vitamin B, E, selenium, zinc, copper, magnesium, and phytochemicals ${ }^{12}$. Whole grains and dietary fiber have long been considered beneficial in lowering the risks of $\mathrm{T}_{2} \mathrm{DM}^{13,14}$, cardiovascular diseases ${ }^{15,16}$, and some types of human cancers ${ }^{17-20}$. Intake of whole grains has also shown a potential beneficial impact on the risk or progression of NAFLD ${ }^{21,22}$. A recent systematic review suggested a protective role for whole grain intake in HCC risk ${ }^{23}$. So far, two prospective cohort studies have reported inverse associations between dietary fiber intake and liver cancer risk ${ }^{24,25}$, and one reported an inverse association for whole grain intake ${ }^{25}$. However, these two studies included a limited number of cases. Specifically, the European Prospective Investigation into Cancer and Nutrition (EPIC) included 191 HCC and 66 ICC, while the Nurses' Health Study (NHS) and the Health Professionals Follow-up Study (HPFS) included 141 HCC cases. No study has yet examined the association between whole grain and dietary fiber intake and risk of CLD death.

In this work, we report inverse associations of whole grain and dietary fiber intake with both incident liver cancer and CLD deaths by utilizing the National Institutes of Health (NIH)-American Association of Retired Persons (AARP) Diet and Health Study with over 900 cases each.

\section{Results}

Baseline characteristics of the cohort participants. A total of 290,484 men (59.8\%) and 195,233 (40.2\%) women were included in this study. The mean age at study entry was 61.5 years old $(\mathrm{SD}=5.4 \mathrm{y})$. The majority of the participants were white $(92.8 \%)$, and $39.8 \%$ had, at least, a college degree or above. Participants in the highest quintile of whole grain intake or total dietary fiber intake compared with those in the lowest quintile were more physically active, had lower alcohol and tobacco consumption, and were more likely to have a self-reported history of diabetes (Table 1). Similar patterns were also observed for men (Supplementary Table 1) and for women (Supplementary Table 2).

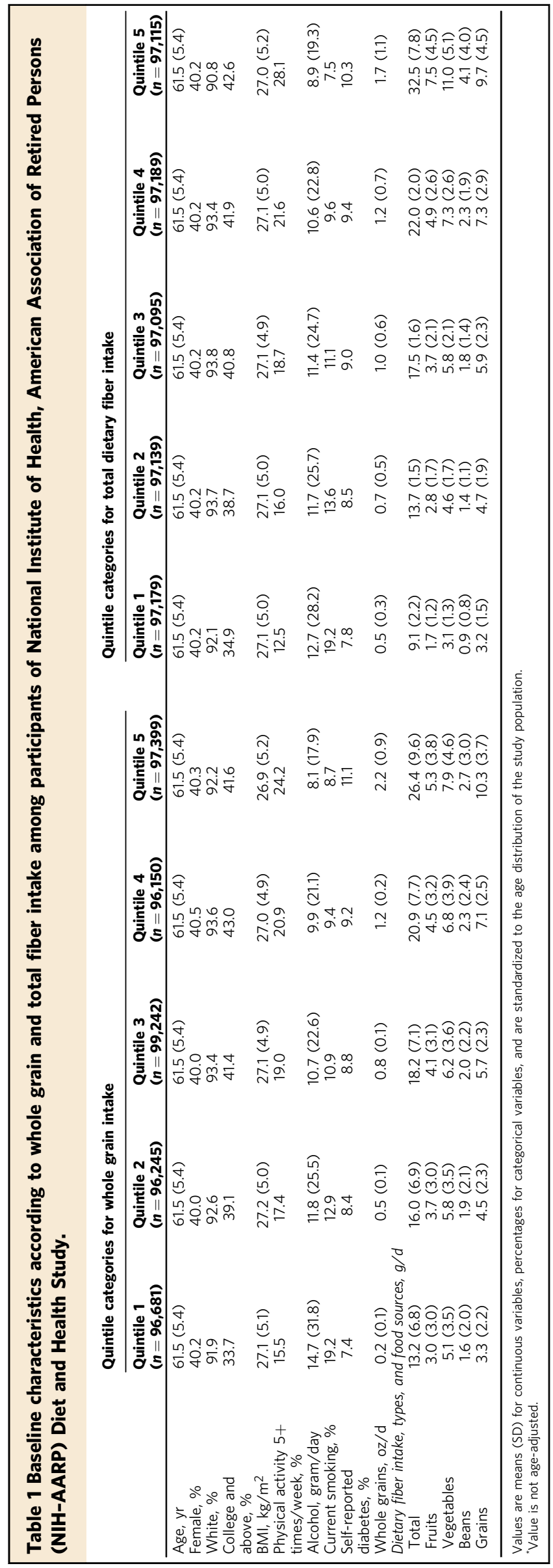


Whole grain, dietary fiber intake, and risk of liver cancer. After a median follow-up time of 15.5 years, a total of 940 participants developed primary liver cancer, including 635 HCC and 164 ICC. Whole grain intake was associated with a $22 \%$ risk reduction of liver cancer $\left(\mathrm{HR}_{\mathrm{Q} 5}\right.$ vs. Q1 $=0.78,95 \% \mathrm{CI}$ : $\left.0.63-0.96, P_{\text {trend }}=0.01\right)$ after multivariable adjustment. Total dietary fiber intake was associated with $31 \%$ risk reduction $\left(\mathrm{HR}_{\mathrm{Q} 5}\right.$ vs. $\mathrm{Q} 1=0.69,0.53-0.90, P_{\text {trend }}<$ 0.001 ) (Table 2). For different sources of fiber, significantly inverse associations were observed for vegetables $\left(\mathrm{HR}_{\mathrm{Q} 5}\right.$ vs. $\mathrm{Q} 1=0.65,95 \%$ CI: $\left.0.52-0.83, P_{\text {trend }}<0.001\right)$ and grains $\left(\mathrm{HR}_{\mathrm{O} 5}\right.$ vs. $\mathrm{Q} 1=0.78,95 \%$ CI: $\left.0.62-0.99, P_{\text {trend }}=0.04\right)$, but not for fruits $\left(\mathrm{HR}_{\mathrm{Q} 5}\right.$ vs. $\mathrm{Q} 1=0.94$, 95\% CI: $\left.0.76-1.17, P_{\text {trend }}=0.82\right)$ or beans $\left(\mathrm{HR}_{\mathrm{Q} 5 \text { vs. } \mathrm{Q} 1}=0.89,95 \%\right.$ CI: $0.71-1.10, P$ trend $=0.02)$. The inverse associations for total dietary fiber and fiber from vegetables were observed for men (Supplementary Table 3), and to a less extent for women (Supplementary Table 4). Significant inverse associations were found between total dietary fiber and fiber from vegetables and risk of HCC (Supplementary Table 5), but not risk of ICC (Supplementary Table 6).

In stratified analyses, the inverse associations of whole grains and risk of liver cancer were generally observed in different subgroups (Fig. 1).

Whole grain, dietary fiber intake, and CLD mortality. A total of 993 CLD deaths were identified. Whole grain intake was associated with a $56 \%$ reduction of CLD mortality $\left(\mathrm{HR}_{\mathrm{Q} 5}\right.$ vs. $\mathrm{Q} 1=0.44$, 95\% CI: $\left.0.35-0.55, P_{\text {trend }}<0.001\right)$ after multivariable adjustment. Total dietary fiber intake was associated with a $63 \%$ reduction of CLD mortality $\left(\mathrm{HR}_{\mathrm{Q} 5}\right.$ vs. $\mathrm{Q} 1=0.37,95 \% \mathrm{CI}: 0.29-0.48, P_{\text {trend }}<$ 0.001) (Table 3). For different sources of fiber, the inverse associations were observed for vegetables $\left(\mathrm{HR}_{\mathrm{Q} 5}\right.$ vs. $\mathrm{Q} 1=0.55,95 \%$ CI: $\left.0.44-0.69, P_{\text {trend }}<0.001\right)$, beans $\left(\mathrm{HR}_{\mathrm{Q} 5 \text { vs. } \mathrm{Q} 1}=0.67,95 \% \mathrm{CI}\right.$ : $\left.0.54-0.84, P_{\text {trend }}=0.03\right)$ and grains $\left(\mathrm{HR}_{\mathrm{Q} 5}\right.$ vs. $\mathrm{Q} 1=0.29,95 \% \mathrm{CI}$ $\left.0.23-0.36, P_{\text {trend }}<0.001\right)$, but not for fruits $\left(\mathrm{HR}_{\mathrm{Q} 5 \mathrm{vs} . \mathrm{Q} 1}=0.98\right.$, 95\% CI: $\left.0.79-1.20, P_{\text {trend }}=0.92\right)$. Similar results were observed in both men (Supplementary Table 7) and women (Supplementary Table 8).

In stratified analyses, the significant inverse associations of whole grain, dietary fiber, and CLD mortality were observed across most subgroups (Fig. 2).

Sensitivity analyses observed similar results. In sensitivity analyses, most of the inverse associations for risk of liver cancer and CLD mortality remained essentially unchanged when we further adjusted for HEI-2015, or excluded cases diagnosed within the first 2 or 5 years of follow-up, or excluded heavy alcohol drinkers.

\section{Discussion}

In this cohort study, participants with the highest quintile of whole grain intake had a $22 \%$ reduced risk of liver cancer and a $56 \%$ reduction in CLD mortality. Participants with the highest quintile of total dietary fiber intake had a $31 \%$ reduced risk of liver cancer and a $63 \%$ reduction in CLD mortality. These inverse associations were observed for fiber from vegetables, beans, and grains but not from fruits.

A recent systematic review reported an inverse association between higher whole grain intake and HCC risk ${ }^{23}$. Thus far, two cohort studies have reported associations between dietary fiber intake and risk of liver cancer. The European Prospective Investigation into Cancer and Nutrition (EPIC) $(n=191$ HCC cases $)$

Table 2 The associations between whole grains and dietary fiber with risk of liver cancer from the NIH-AARP Diet and Health Study.

HR $(95 \% \mathrm{Cl})$

$P$ trend

\begin{tabular}{|c|c|c|c|c|c|c|c|}
\hline & Quintile 1 & Quintile 2 & Quintile 3 & Quintile 4 & Quintile 5 & Per SD increase & \\
\hline \multicolumn{8}{|l|}{ Whole grains } \\
\hline Case number & 214 & 194 & 198 & 155 & 179 & & \\
\hline Model 1 & 1 (ref) & $0.88(0.72,1.07)$ & $0.86(0.71,1.04)$ & $0.68(0.56,0.84)$ & $0.77(0.64,0.95)$ & $0.91(0.84,0.97)$ & 0.007 \\
\hline Model 2 & 1 (ref) & $0.91(0.74,1.10)$ & $0.91(0.75,1.11)$ & $0.73(0.59,0.90)$ & $0.78(0.63,0.96)$ & $0.90(0.83,0.97)$ & 0.005 \\
\hline \multicolumn{8}{|c|}{ Total dietary fiber } \\
\hline Case number & 193 & 210 & 177 & 187 & 173 & & \\
\hline Model 1 & 1 (ref) & $1.06(0.87,1.29)$ & $0.88(0.72,1.08)$ & $0.92(0.75,1.12)$ & $0.84(0.69,1.04)$ & $0.90(0.83,0.98)$ & 0.016 \\
\hline Model 2 & 1 (ref) & $1.04(0.85,1.27)$ & $0.83(0.67,1.04)$ & $0.84(0.67,1.05)$ & $0.69(0.53,0.90)$ & $0.81(0.72,0.90)$ & $<0.001$ \\
\hline \multicolumn{8}{|l|}{ Fiber from fruits } \\
\hline Case number & 185 & 197 & 186 & 185 & 187 & & \\
\hline Model 1 & 1 (ref) & $1.00(0.82,1.23)$ & $0.93(0.76,1.14)$ & $0.90(0.73,1.11)$ & $0.90(0.74,1.11)$ & $0.99(0.92,1.06)$ & 0.760 \\
\hline Model 2 & 1 (ref) & $1.06(0.87,1.30)$ & $1.00(0.82,1.24)$ & $0.98(0.79,1.20)$ & $0.94(0.76,1.17)$ & $0.99(0.92,1.07)$ & 0.822 \\
\hline \multicolumn{8}{|c|}{ Fiber from vegetables } \\
\hline Case number & 211 & 186 & 202 & 180 & 161 & & \\
\hline Model 1 & 1 (ref) & $0.87(0.72,1.07)$ & $0.95(0.78,1.15)$ & $0.85(0.69,1.03)$ & $0.76(0.62,0.93)$ & $0.89(0.82,0.97)$ & 0.008 \\
\hline Model 2 & 1 (ref) & $0.89(0.73,1.08)$ & $0.94(0.77,1.14)$ & $0.80(0.65,0.99)$ & $0.65(0.52,0.83)$ & $0.82(0.74,0.91)$ & $<0.001$ \\
\hline \multicolumn{8}{|l|}{ Fiber from beans } \\
\hline Case number & 198 & 197 & 203 & 153 & 189 & & \\
\hline Model 1 & 1 (ref) & $0.98(0.81,1.20)$ & $1.03(0.85,1.25)$ & $0.77(0.62,0.95)$ & $0.96(0.78,1.17)$ & $0.95(0.88,1.03)$ & 0.214 \\
\hline Model 2 & 1 (ref) & $1.01(0.83,1.23)$ & $1.05(0.86,1.28)$ & $0.77(0.62,0.96)$ & $0.89(0.71,1.10)$ & $0.90(0.83,0.99)$ & 0.023 \\
\hline \multicolumn{8}{|l|}{ Fiber from grains } \\
\hline Case number & 196 & 205 & 169 & 198 & 172 & & \\
\hline Model 1 & 1 (ref) & $1.02(0.84,1.24)$ & $0.83(0.68,1.02)$ & $0.97(0.79,1.18)$ & $0.82(0.67,1.01)$ & $0.93(0.86,1.00)$ & 0.055 \\
\hline Model 2 & 1 (ref) & $1.02(0.83,1.24)$ & $0.82(0.67,1.02)$ & $0.95(0.77,1.17)$ & $0.78(0.62,0.99)$ & $0.91(0.84,0.99)$ & 0.037 \\
\hline
\end{tabular}

The SDs of intake were $0.86 \mathrm{oz} /$ day for whole grains, $10.9 \mathrm{~g} /$ day for total dietary fiber, $3.6 \mathrm{~g} /$ day for fiber from fruits, $4.8 \mathrm{~g} /$ day for fiber from vegetables, $2.9 \mathrm{~g} /$ day for fiber from beans, and $4.0 \mathrm{~g} / \mathrm{day}$ for fiber from grains.

Cox proportional hazard regression models were used. All $P$-values were two-sided. Model 1 were stratified by sex, adjusted for age at baseline (continuous). Model 2 further adjusted for level of education (' $\leq 11$ years', 'high school', 'vocational technology school', 'some college', 'college/postgraduate'), race ('non-Hispanic white', 'non-Hispanic black', 'Hispanic', 'Asian, Pacific Islander, or American Indian/Alaskan native'), BMI ('<25', '25-30', '30+' kg/m²), alcohol use ('non-drinker', '0.1-4.9', '5-9.9', '10+', gram/day), tobacco smoking ('never smoked', 'former smoker', 'current smoker'), physical activity ('never', 'rarely', '1-3 time per month', '1-2 times per week', '3-4 times per week', '5 + times per week'), history of diabetes ('no', 'yes') and total energy intake (continuous). 


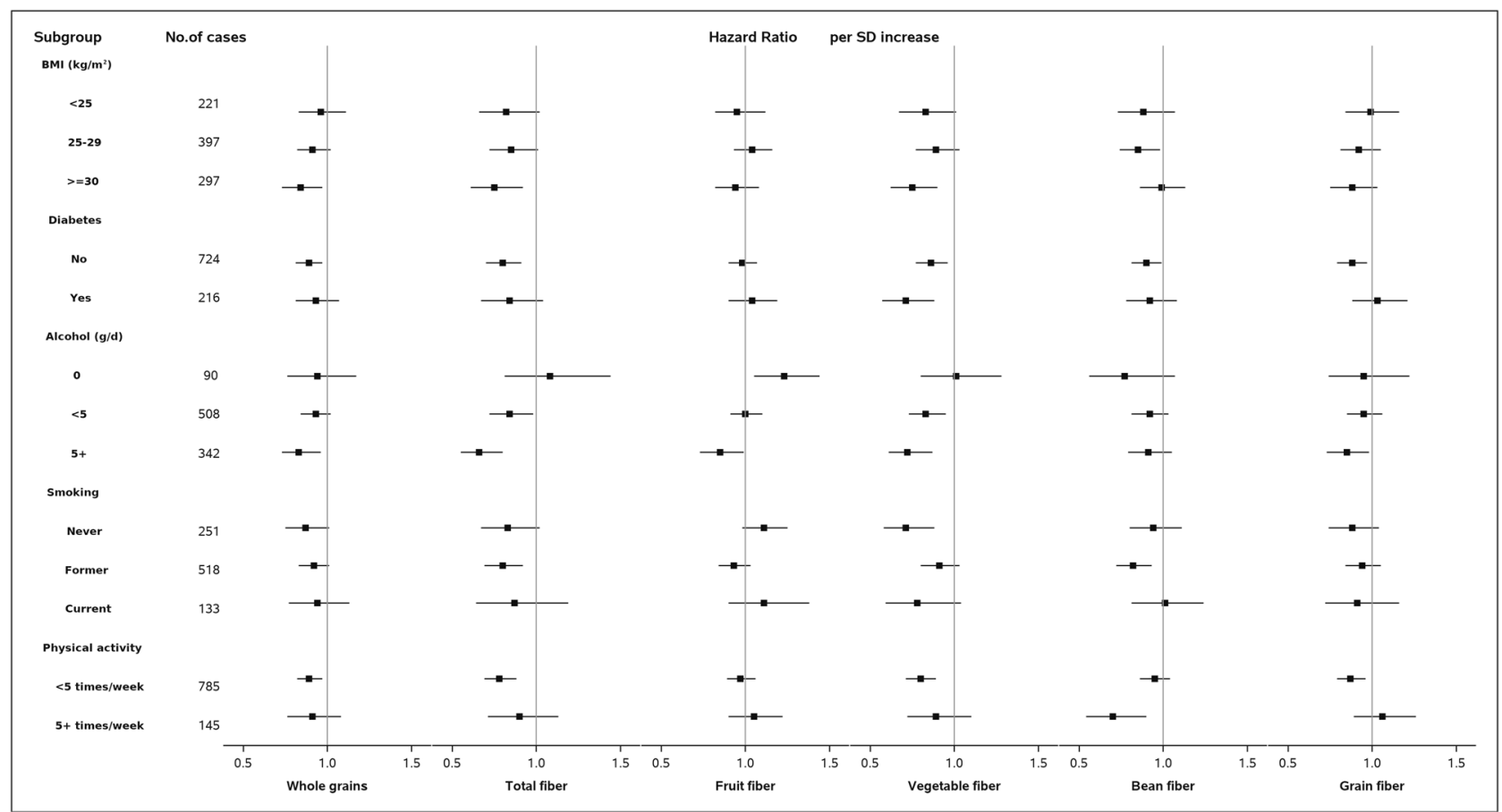

Fig. 1 Stratified analyses for association between whole grain and dietary fiber intake with risk of liver cancer among the participants of NIH-AARP Diet and Health Study. Cox proportional hazard regression models were used. All P-values were two-sided. Models were stratified by sex and adjusted for age at baseline (continuous), level of education (' $\leq 11$ years', 'high school', 'vocational technology school', 'some college', 'college/postgraduate'), race ('non-Hispanic white', 'non-Hispanic black', 'Hispanic', 'Asian, Pacific Islander, or American Indian/Alaskan native'), BMI ('<25', '25-30', '30 +' kg/m²), alcohol use ('Non-drinker', '0.1-4.9', '5-9.9', '10 +' g/day), tobacco smoking ('never smoked', 'former smoker', 'current smoker'), physical activity ('never', 'rarely', '1-3 time per month', '1-2 times per week', '3-4 times per week', '5+ times per week'), history of diabetes ('no', 'yes') and total energy intake (continuous), except when the variable is used for stratification. The $P$ for interaction between whole grain or fiber intake and characteristics for stratification in risk of liver cancer were as follows: Whole grain: 0.22 for BMI, 0.25 for diabetes, 0.20 for alcohol use, 0.42 for smoking, and 0.98 for physical activity. Total fiber: 0.87 for BMI, 0.18 for diabetes, 0.02 for alcohol use, 0.76 for smoking, and 0.53 for physical activity. Fiber from fruits: 0.71 for BMI, 0.36 for diabetes, $<0.01$ for alcohol use, 0.41 for smoking, and 0.29 for physical activity. Fiber from vegetables: 0.68 for BMI, 0.54 for diabetes, 0.05 for alcohol use, 0.37 for smoking, and 0.57 for physical activity. Fiber from beans: 0.15 for BMI, 0.33 for diabetes, 0.35 for alcohol use, 0.63 for smoking, and 0.02 for physical activity. Fiber from grains: 0.69 for BMI, 0.02 for diabetes, 0.44 for alcohol use, 0.51 for smoking, and 0.23 for physical activity. Abbreviations: BMI body mass index; SD standard deviation; NIH-AARP: National Institutes of Health-American Association of Retired Persons.

reported significant associations for total fiber $\left(\mathrm{HR}_{\mathrm{Q} 4}\right.$ vs. Q1 $=0.51$, 95\% CI: 0.31-0.83) and fiber from cereals, but not from vegetables, fruits, and other sources ${ }^{24}$. The Nurses' Health Study (NHS) and the Health Professionals Follow-up Study (HPFS) $(n=141$ HCC cases) reported a suggestive association between fiber from cereals $\left(\mathrm{HR}_{\mathrm{T} 3}\right.$ vs. T1 $\left.=0.68,95 \% \mathrm{CI}: 0.45-1.03, P_{\text {trend }}=0.07\right)$, but not fiber from fruits or vegetables and HCC risk ${ }^{25}$. Moreover, the NHS/ HPFS study also found an inverse association between whole grain intake and $\mathrm{HCC}$ risk $\left(\mathrm{HR}_{\mathrm{T} 3} \text { vs. } \mathrm{T} 1=0.63,95 \% \mathrm{CI} \text { : } 0.41 \text { to } 0.96\right)^{25}$. In this current study, no association was found for fiber from fruits, and an inverse association was found for whole grains and total fiber, which were consistent with the observation from the EPIC $^{24}$ and NHS/HPFS studies ${ }^{25}$. Moreover, inverse associations were observed for fiber from vegetables and grains in this study, adding to the knowledge that fiber from different food sources might have different associations with liver cancer. Furthermore, this study reports the inverse associations between whole grains, total dietary fibers, fibers from vegetables, and grains with CLD mortality, expanding the potential benefit of whole grain and dietary fiber intake from the risk of liver cancer to a wide range of chronic liver diseases.

The biologic mechanisms for the inverse associations of whole grains and dietary fiber with the liver disease remain to be fully elucidated. Whole grains are good sources of dietary fiber, resistant starch, and oligosaccharides, which may increase stool bulk, speed intestinal transit and reduce the exposure to carcinogens in the colon ${ }^{35}$. Whole grains contain antioxidants and phytoestrogens and have been reported to have a protective effect among certain gastrointestinal cancers and hormonesensitive cancers ${ }^{36}$. Dietary fiber has been reported to have beneficial effects on conditions related to liver disease and liver cancer, including blood glucose ${ }^{37}$, insulin sensitivity ${ }^{37}$, liver fat content $^{38}$, and metabolic syndrome ${ }^{39-41}$. Oligosaccharides as fermentable carbohydrates are fermented in the colon and increase the production of short-chain fatty acids, which are important in the maintenance of gut integrity and regulation of inflammation ${ }^{36,42}$. Recent studies discovered that the effects of dietary fiber might be mediated by the alteration in gut microbiota, such as an increased abundance of Prevotella, Treponema, and Succinivibrio ${ }^{43-45}$. Moreover, increasing evidence revealed the existence of a gut-liver axis, which exchanges signals between the bowel and liver. The portal vein allows transporting products generated by microbial communities to the liver, and the microbial communities are critical in the homeostasis of the gut-liver axis. Given that evidence accumulates for the pathogenetic role of microbe-derived metabolites in NAFLD, a similar mechanism may apply to more advanced liver diseases ${ }^{46}$. Our results also found heterogeneous associations for fiber from different food sources. Beans, vegetables, and fruits are good sources of dietary fiber. Interestingly, fiber from fruits did not show an inverse association with liver disease mortality, as did fiber from other sources. Although the underlying mechanism was unclear, 
Table 3 The associations between whole grains and dietary fiber intake with CLD mortality from the NIH-AARP Diet and Health Study.

HR (95\% CI)

$P$ trend

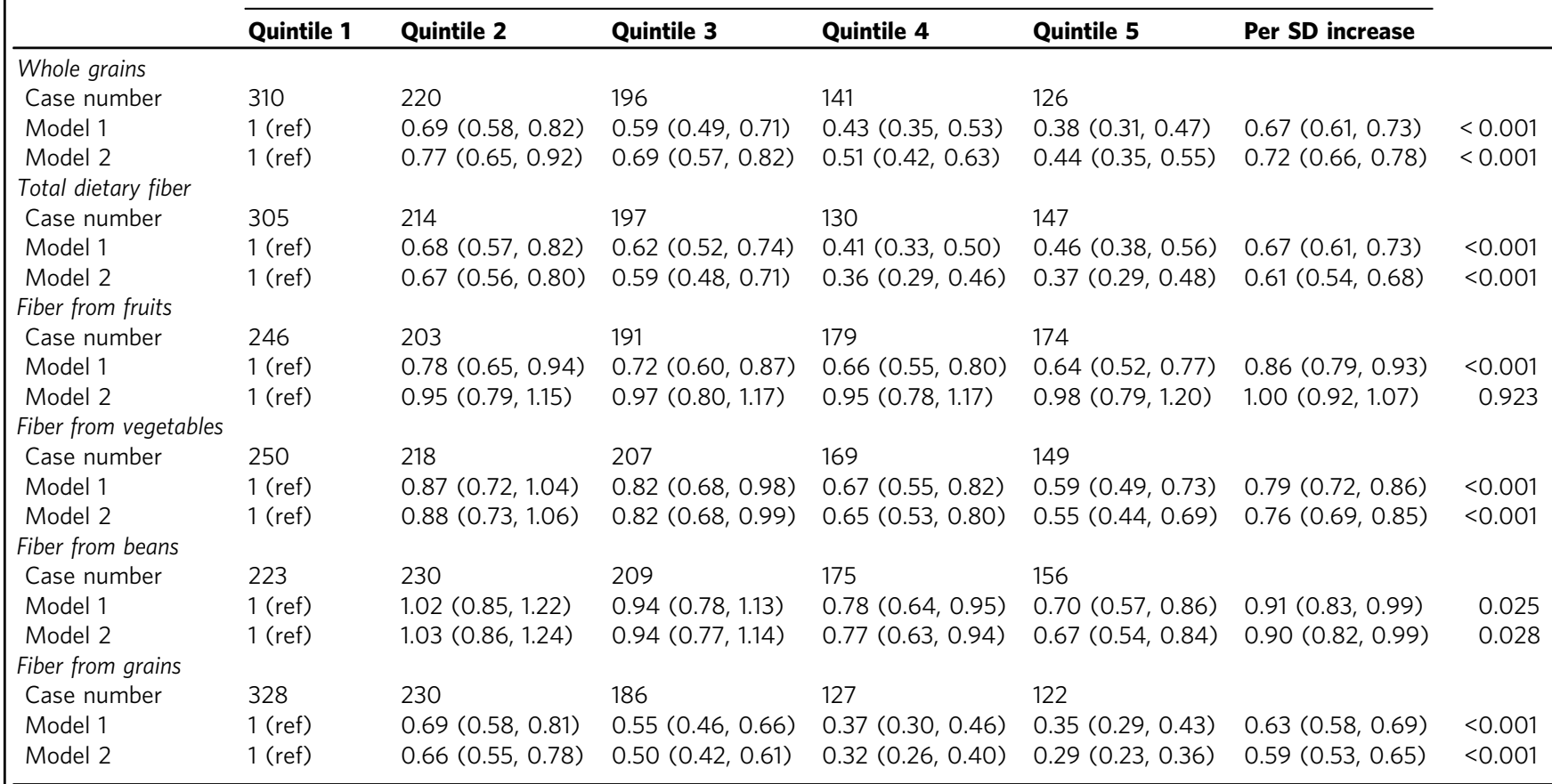

The SDs of intake were $0.86 \mathrm{oz} /$ day for whole grains, $10.9 \mathrm{~g} /$ day for total dietary fiber, $3.6 \mathrm{~g} /$ day for fiber from fruits, $4.8 \mathrm{~g} /$ day for fiber from vegetables, $2.9 \mathrm{~g} /$ day for fiber from beans, and $4.0 \mathrm{~g} / \mathrm{day}$ for fiber from grains.

Cox proportional hazard regression models were used. All P-values were two-sided. Model 1 were stratified by sex, adjusted for age at baseline (continuous). Model 2 further adjusted for level of education (' $\leq 11$ years', 'high school', 'vocational technology school ', 'some college', 'college/postgraduate'), race ('non-Hispanic white', 'non-Hispanic black', 'Hispanic', 'Asian, Pacific Islander, or American Indian/Alaskan native'), BMI ('<25', '25-30', '30+', kg/m²), alcohol use ('Non-drinker', '0.1-4.9', '5-9.9', '10+',gram/day), tobacco smoking ('never smoked', 'former smoker', 'current smoker'), physical activity ('never', 'rarely', '1-3 time per month', '1-2 times per week', '3-4 times per week', '5+ times per week'), history of diabetes ('no', 'yes') and total energy intake (continuous).

excess fructose has been reported to be associated with increased insulin resistance, fatty acid production, oxidative stress, and fatty liver ${ }^{47}$. Fruits high in fructose might cancel out the beneficial effect of fiber due to an adverse effect of fructose ${ }^{48}$. Collectively, our study suggests that the inverse association between dietary fiber and liver disease mortality may vary depending on the source of fiber. More investigation is needed to further clarify the potential mechanisms.

The present study is the largest cohort study to date to evaluate the associations between whole grain and dietary fiber intake with liver cancer risk. In addition, it reports an association between dietary fiber intake and CLD mortality. With the large sample size, dietary fiber from different food sources was examined in detail, and two major subtypes of primary liver cancer (i.e., HCC and ICC) were studied. Many of the important lifestyle confounders for liver cancer and CLD, including age, alcohol, tobacco use, obesity, and diabetes, were included in the analyses. However, there are several limitations to this study. First, as important risk factors for liver cancer and CLD, HBV/HCV was not measured in the full cohort, neither was information on history of hepatitis collected. It is estimated that the prevalence is $0.1 \%$ for $\mathrm{HBV}$ and $1.0 \%$ for $\mathrm{HCV}$ in the US population ${ }^{49,50}$. Using the data from the Liver Cancer Pooling Project and NHS/ HPFS cohorts, we and others have reported no correlations between $\mathrm{HBV} / \mathrm{HCV}$ infections with $\mathrm{BMI}^{51}$, smoking ${ }^{52}$, alcohol use $^{52}$, coffee intake ${ }^{53}$, fiber ${ }^{25}$, nuts ${ }^{54}$, fatty acids ${ }^{55}$, dairy foods ${ }^{56}$, or meats ${ }^{57}$. Furthermore, previous studies have reported similar findings with or without adjustment for $\mathrm{HBV} / \mathrm{HCV}$ for associations of liver cancer risk with tooth loss ${ }^{58}$, fish intake $e^{59}$, and coffee $^{60}$. Thus, the relationship between diet and liver cancer risk in this study is unlikely to be substantially confounded by HBV/
HCV infection status. Second, whole grain and dietary fiber intake were derived from the self-reported FFQ. The FFQ was validated by using two non-consecutive 24 -h dietary recalls. The energy-adjusted correlation coefficients for dietary fiber intake were 0.72 among men and 0.66 among women ${ }^{31}$. Measurement errors cannot be ruled out. We examined the associations for dietary fiber and whole grain based on ranking instead of absolute intake levels, because the FFQ is better suited to ranking according to relative intake than assessing precise intake quantitatively. Third, the food intake questionnaire was selfadministered at baseline. Thus, the change of diet during the follow-up was not recorded or analyzed. We cannot evaluate the associations between changes of whole grain and dietary fiber intake with the outcomes. Fourth, people who had higher whole grain or fiber intake were more likely to have a healthier diet and lifestyle overall, which might contribute to the reduced risk. Residual confounding might exist. Fifth, in this analysis, the outcomes of interest were liver cancer incidence and CLD mortality. While it would have been ideal for examining CLD incidence in addition to morality, CLD registries do not exist in the US, nor is there a single medical records system to permit the identification of CLD diagnoses. As a result, CLD mortality was analyzed in the current study. Finally, the study participants are predominantly persons of European ancestry and are individuals aged 50-71 years old at the beginning of the follow-up, which potentially limited the generalizability of the results to other populations.

In conclusion, we found significant inverse associations between whole grain and dietary fiber intake with liver cancer incidence and CLD mortality in a US population. The associations between dietary fiber were particular for fiber from 


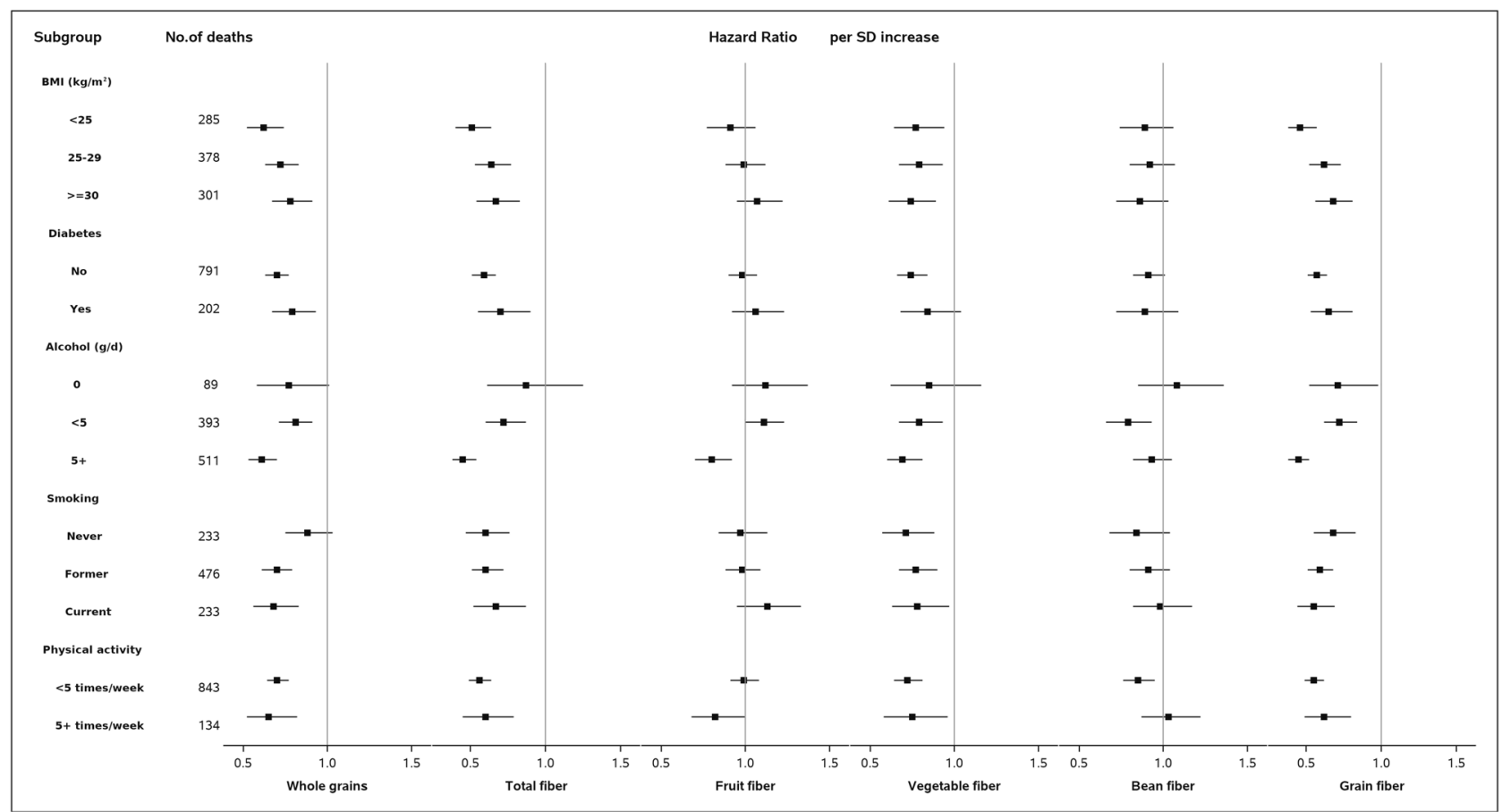

Fig. 2 Stratified analyses for association between whole grain and dietary fiber intake with CLD mortality among the participants of NIH-AARP Diet and Health Study. Cox proportional hazard regression models were used. All P-values were two-sided. Models were stratified by sex and adjusted for age at baseline (continuous), level of education (' $\leq 11$ years', 'high school', 'vocational technology school ', 'some college', 'college/postgraduate'), race ('nonhispanic white', 'non-hispanic black', 'hispanic', 'asian, Pacific Islander, or American Indian/Alaskan native'), BMI ('<25', '25-30', '30 +', kg/m²), alcohol use ('non-drinker', '0.1-4.9', '5-9.9', '10 +', gram/day), tobacco smoking ('never smoked', 'former smoker', 'current smoker'), physical activity ('never', 'rarely', '1-3 time per month', '1-2 times per week', '3-4 times per week', '5 + times per week'), history of diabetes ('no', 'yes') and total energy intake (continuous), except when the variable is used for stratification. The $P$ for interaction between whole grain or fiber intake and characteristics for stratification in CLD mortality were as follows: Whole grain: 0.01 for $\mathrm{BMl}, 0.07$ for diabetes, $<0.01$ for alcohol use, $<0.01$ for smoking, and 0.73 for physical activity. Total fiber: 0.03 for BMI, 0.05 for diabetes, $<0.01$ for alcohol use, 0.96 for smoking, and 0.52 for physical activity. Fiber from fruits: $<0.01$ for BMI, 0.10 for diabetes, $<0.01$ for alcohol use, 0.97 for smoking, and 0.16 for physical activity. Fiber from vegetables: 0.83 for BMI, 0.11 for diabetes, 0.72 for alcohol use, 0.63 for smoking, and 0.76 for physical activity. Fiber from beans: 0.87 for BMI, 0.84 for diabetes, 0.23 for alcohol use, 0.07 for smoking, and 0.07 for physical activity. Fiber from grains: $<0.01$ for BMl, 0.13 for diabetes, $<0.01$ for alcohol use, 0.15 for smoking, and 0.40 for physical activity. Abbreviations: BMI body mass index; SD standard deviation; NIH-AARP National Institutes of Health-American Association of Retired Persons.

vegetables, beans, and grains. These findings, if confirmed by future studies, indicate that a diet rich in whole grain and dietary fiber could be recommended for reducing liver cancer incidence and CLD mortality. Further studies are warranted to clarify the underlying mechanisms and determine specific dietary components associated with benefit in diverse racial/ethnic populations.

\section{Methods}

Study population. The NIH-AARP Diet and Health Study recruited men and women aged 50-71 years old by mailing questionnaires to its 3.5 million members from six US states (California, Florida, Louisiana, New Jersey, North Carolina, and Pennsylvania) and two metropolitan areas (Atlanta, GA and Detroit, MI) in $1995-1996^{26}$. A total of 566,398 persons (339,666 men and 226,732 women) returned the baseline questionnaire and were included in the final cohort. In this analysis, we excluded participants with: (1) prevalent cancer $(n=50,118)$ at baseline; (2) extreme caloric intakes $(<500$, or $>3,500$ for women; $<800$, or $>4,000$ for men; $n=29,983$ ); and (3) participants who were diagnosed with liver cancer or died from CLD before their baseline survey questionnaires were scanned $(n=580)$. Thus, the analytic cohort included 485,717 (290,484 men and 195,233 women). The study was approved by the Special Studies Institutional Review Board of the U.S. National Cancer Institute. Informed consent has been obtained from all participants.

\section{Case ascertainment}

Liver cancer. Cases of primary liver cancer (defined by International Classification of Diseases, 10th edition [ICD-10] diagnostic code C22) were identified by linkage with state cancer registries through December $31^{\text {st }}, 2011^{26}$. Cases of HCC were further defined by morphology codes $8170-8175$. Cases of ICC were defined by morphology codes $8032,8033,8070,8071,8140,8141,8160,8161,8260,8480$,
8481,8490 and $8560^{27}$. Prior examination reported that these codes have a sensitivity of $90 \%$ and a specificity of nearly $100 \%$ for cancer identification ${ }^{28}$.

Deaths due to chronic liver diseases. The National Death Index Plus (NDIP) was used to identify causes of death through December $31^{\text {st }}, 2011$. Consistent with previous studies in the same cohort ${ }^{28,29}$, deaths due to chronic liver diseases included deaths from fibrosis, cirrhosis, alcoholic liver diseases, and chronic hepatitis (ICD-9: 571.0, 571.2-571.6, 571.8, and 571.9; ICD-10: K70, K73, and K74). Classification of CLD from the NDIP was validated using the electronic medical records from members of the Kaiser Permanente Medical Care Program of Northern California, and specificity was found to be $89 \%{ }^{30}$.

Dietary assessment. The information on diet was collected via a 124 -item foodfrequency questionnaire (FFQ) ${ }^{26}$. The FFQ was validated among 2,000 NIH-AARP participants using two 24 -h dietary recalls and a second FFQ ${ }^{26,31}$. The energyadjusted correlation coefficients for dietary fiber intake were 0.72 in men and 0.66 in women ${ }^{31}$. Food groups were created with the MyPyramid Equivalents Database (MPED) version 1.0, and component ingredients were disaggregated from food mixtures, assigned to food groups, and calculated to cup or ounce equivalents $\mathrm{s}^{32}$. Nutrients were calculated with the 1994-1996 U.S. Department of Agriculture's Continuing Survey of Food Intakes by Individuals (CSFII) ${ }^{33}$. The assessments of whole grain and fiber intake were described in the previous study from the same cohort $^{34}$. Specifically, whole grains were defined and extracted as the whole grain part of each food item, including ready-to-eat cereals, high-fiber cereals, cooked cereals, other fiber cereals, whole-grain bread or dinner rolls, popcorn, pancakes, waffles, French toast or crepes, rice, or other cooked grains, bagels, English muffins, tortillas, pasta, crackers, chips, cookies, or brownies, sweet pastries, and pies. Whole grain intake was presented as MPED number of whole grains in ounce equivalents per day (oz eq/day). The USDA's Pyramid Servings Database was used to estimate whole grain intake from all foods collected in the FFQ, even from those foods with small amounts of whole grain. Total fiber from fruits, vegetables, beans and grains 
was estimated by summing dietary fiber from these foods. In the questionnaire, the participants were asked about the frequency and amount of intake for different kinds of vegetables and fruits. They were also asked about beans including 'beans, such as baked beans, refried beans, pintos, kidney, lima, lentils, or soybeans (not including bean soup)' and 'bean-based soups'. For fruits and vegetables, a 1-cup equivalent is defined as 1-cup raw or cooked fruits or vegetables, 1-cup fruit or vegetable juice, 0.5 cup dried fruits, or 2-cups leafy greens. For beans, a 1-cup equivalent is defined as 1-cup cooked dry beans or peas. For grains, a 1-ounce equivalent is defined as one regular slice of yeast bread, or 0.5-cup rice, pasta, or cooked cereal, or 1 cup ready-to-eat cereal. The equivalents were further converted into gram amounts 32

Covariate assessment. Socio-demographic characteristics including age, race (self-reported), weight, height, level of education, and other potential risk factors including tobacco smoking, alcohol drinking, physical activity, self-reported history of diabetes were also collected in the baseline questionnaire ${ }^{26}$. Participants reported their highest level of education from the following categories: <8 years; 8-11 years; 12 years, or completed high school; post-high school training other than college (e.g., vocational or technical training); some college; college graduate; postgraduate. The frequency and amount of reported alcohol consumption (including beer, wine, or wine coolers, and liquor or mixed drinks) were converted to grams per day in analyses. Participants reported whether they had ever smoked 100 or more cigarettes during lifetime and if they currently smoked or had stopped. Frequency (never, rarely, 1-3 times per month, 1-2 times per week, 3-4 times per week, 5+ times per week) in the past 12 months of physical activity at work or home (including exercise, sports, and activities such as carrying heavy loads) $\geq$ 20 minutes that caused increases in breathing or heart rate or sufficient to work up a sweat was asked.

Statistical analyses. For liver cancer, person-years to the event was calculated for each participant from baseline to date of diagnosis of primary liver cancer, date of death, or end of follow-up (December $31^{\mathrm{st}}, 2011$ ), whichever came the first. For CLD mortality, person-years to the event was calculated from baseline to date of CLD death, date of death from other reasons, or end of follow-up (December $31^{\text {st }}$, 2011), whichever came the first. Cox proportional hazard regression models were used to estimate hazard ratios (HRs) and 95\% confidence intervals (CIs) for events comparing across quintile categories of whole grain or dietary fiber intake with the lowest intake quintile as the reference group. The proportional hazards assumption was tested using a cross-product term of $\log$ (time) and whole grain or dietary fiber intake; no violations were observed $(P>0.05) . P$ for trend was calculated per SD increase in whole grain or dietary fiber intake. The associations of whole grain and fiber intake with liver cancer incidence and CLD mortality were not modified by sex $(P$ for heterogeneity $>0.05)$. Therefore, we report pooled estimates, with sexspecific results presented in Supplementary Tables.

Multivariable models included the following a priori covariates: age at baseline (continuous), level of education ( $\leq 11$ years; high school; vocational technology school; some college; college/postgraduate), race (non-Hispanic white; nonHispanic black; Hispanic; Asian, Pacific Islander, or American Indian/Alaskan Native), body mass index (BMI, $<25 ; 25-30 ; \geq 30 \mathrm{~kg} / \mathrm{m}^{2}$ ), alcohol consumption (non-drinker; $0.1-4.9 ; 5-9.9 ; \geq 10 \mathrm{~g} /$ day), tobacco smoking (never smoked; former smoker; current smoker), physical activity (never; rarely; 1-3 time per month; 1-2 times per week; 3-4 times per week; 5 or more times per week), self-reported history of diabetes (no; yes), and total energy intake (continuous).

Subgroup analyses were conducted across categories of BMI, self-reported history of diabetes, alcohol drinking, tobacco smoking, and physical activity. $P$ for interaction was tested by introducing a product term of the two variables examined in the regression models.

In sensitivity analyses, the healthy eating index (HEI-2015) was added to the multivariable regression models to control for overall dietary quality. Second, we excluded cases diagnosed within the first 2 or 5 years of follow-up to address the possible reverse causation. We also excluded heavy alcohol drinkers, defined as men who consumed more than 4 drinks and women who consumed more than 3 drinks per day, to further control for confounding from alcohol drinking.

SAS 9.4 software was used for all analyses (SAS Institute Inc., Cary, NC, USA). All $P$-values were two-sided.

Reporting Summary. Further information on research design is available in the Nature Research Reporting Summary linked to this article.

\section{Data availability statement}

Data used in this study are maintained by the National Cancer Institute, Division of Cancer Epidemiology and Genetics, and are available upon submitting a proposal to be approved by the NIH-AARP Steering Committee for privacy concerns. For more information, visit https://www.nihaarpstars.com/.

\section{Code availability statement}

Code used in this study is available; please contact the corresponding author.
Received: 22 January 2021; Accepted: 27 September 2021; Published online: 04 November 2021

\section{References}

1. Bray, F. et al. Global cancer statistics 2018: GLOBOCAN estimates of incidence and mortality worldwide for 36 cancers in 185 countries. CA Cancer J. Clin. 68, 394-424 (2018).

2. Ryerson, A. B. et al. Annual report to the nation on the status of cancer, 1975 2012, featuring the increasing incidence of liver cancer. Cancer 122, 1312-1337 (2016)

3. Lee, Y.-T. et al. The mortality and overall survival trends of primary liver cancer in the United States. J Natl Cancer Inst. https://doi.org/10.1093/jnci/ djab079 (2021).

4. Altekruse, S. F., Devesa, S. S., Dickie, L. A., McGlynn, K. A. \& Kleiner, D. E. Histological classification of liver and intrahepatic bile duct cancers in SEER registries. J. Regist Manag. 38, 201-205 (2011)

5. Petrick, J. L. et al. International trends in liver cancer incidence, overall and by histologic subtype, 1978-2007. Int J. Cancer 139, 1534-1545 (2016).

6. Baecker, A., Liu, X., La Vecchia, C. \& Zhang, Z.-F. Worldwide incidence of hepatocellular carcinoma cases attributable to major risk factors. Eur. J. Cancer Prev. J. Eur. Cancer Prev. Organ ECP 27, 205-212 (2018).

7. Makarova-Rusher, O. V. et al. Population attributable fractions of risk factors for hepatocellular carcinoma in the United States. Cancer 122, 1757-1765 (2016).

8. Clements, O., Eliahoo, J., Kim, J. U., Taylor-Robinson, S. D. \& Khan, S. A. Risk factors for intrahepatic and extrahepatic cholangiocarcinoma: A systematic review and meta-analysis. J. Hepatol. 72, 95-103 (2020).

9. Tsochatzis, E. A., Bosch, J. \& Burroughs, A. K. Liver cirrhosis. Lancet Lond. Engl. 383, 1749-1761 (2014).

10. World Cancer Research Fund, American Institute for Cancer Research. Diet, Nutrition, Physical Activity and Cancer: A Global Perspective. (Continuous Update Project Expert Report 2018. Accessed June 19, 2021); https:// www.wcrf.org/wp-content/uploads/2021/02/Summary-of-Third-ExpertReport-2018.pdf.

11. Romaguera, D. et al. Is concordance with world cancer research fund/ American institute for cancer research guidelines for cancer prevention related to subsequent risk of cancer? results from the EPIC study. Am. J. Clin. Nutr. 96, 150-163 (2012).

12. Slavin, J. L., Jacobs, D., Marquart, L. \& Wiemer, K. The role of whole grains in disease prevention. J. Am. Diet. Assoc. 101, 780-785 (2001).

13. Aune, D., Norat, T., Romundstad, P. \& Vatten, L. J. Whole grain and refined grain consumption and the risk of type 2 diabetes: a systematic review and dose-response meta-analysis of cohort studies. Eur. J. Epidemiol. 28, 845-858 (2013).

14. McRae, M. P. Dietary fiber intake and type 2 diabetes mellitus: an umbrella review of meta-analyses. J. Chiropr. Med 17, 44-53 (2018).

15. Threapleton, D. E. et al. Dietary fibre intake and risk of cardiovascular disease: systematic review and meta-analysis. BMJ. 347, https://doi.org/10.1136/ bmj.f6879 (2013).

16. Aune, D. et al. Whole grain consumption and risk of cardiovascular disease, cancer, and all cause and cause specific mortality: systematic review and doseresponse meta-analysis of prospective studies. BMJ. https://doi.org/10.1136/ bmj.i2716 (2016).

17. Park, Y. et al. Dietary fiber intake and risk of colorectal cancer: a pooled analysis of prospective cohort studies. JAMA 294, 2849-2857 (2005).

18. Murphy, N. et al. Dietary fibre intake and risks of cancers of the colon and rectum in the European prospective investigation into cancer and nutrition (EPIC). PloS One 7, e39361 (2012).

19. Chen, S. et al. Dietary fibre intake and risk of breast cancer: a systematic review and meta-analysis of epidemiological studies. Oncotarget 7, 80980-80989 (2016)

20. Zheng, B., Shen, H., Han, H., Han, T. \& Qin, Y. Dietary fiber intake and reduced risk of ovarian cancer: a meta-analysis. Nutr. J. 17, 99 (2018).

21. Ross, A. B., Godin, J.-P., Minehira, K. \& Kirwan, J. P. Increasing whole grain intake as part of prevention and treatment of nonalcoholic Fatty liver disease. Int J. Endocrinol. 2013, 585876 (2013).

22. Georgoulis, M. et al. The impact of cereal grain consumption on the development and severity of non-alcoholic fatty liver disease. Eur. J. Nutr. 53, 1727-1735 (2014)

23. George, E. S. et al. The association between diet and hepatocellular carcinoma: a systematic review. Nutrients 13, 172 (2021).

24. Fedirko, V. et al. Glycemic index, glycemic load, dietary carbohydrate, and dietary fiber intake and risk of liver and biliary tract cancers in Western Europeans. Ann. Oncol. J. Eur. Soc. Med Oncol. 24, 543-553 (2013). 
25. Yang, W. et al. Association of intake of whole grains and dietary fiber with risk of hepatocellular carcinoma in US Adults. JAMA Oncol. 5, 879-886 (2019).

26. Schatzkin, A. et al. Design and serendipity in establishing a large cohort with wide dietary intake distributions: the National Institutes of Health-American Association of Retired Persons Diet and Health Study. Am. J. Epidemiol. 154, 1119-1125 (2001).

27. Fritz, A. G., ed. International Classification of Diseases for Oncology: ICD-O. 3rd edn, (World Health Organization; 2013).

28. Li, W.-Q. et al. Index-based dietary patterns and risk of incident hepatocellular carcinoma and mortality from chronic liver disease in a prospective study. Hepatol. Balt. Md. 60, 588-597 (2014).

29. Kung, H.-C., Hoyert, D. L., Xu, J. \& Murphy, S. L. Deaths: final data for 2005. Natl Vital-. Stat. Rep. Cent. Dis. Control Prev. Natl Cent. Health Stat. Natl Vital-. Stat. Syst. 56, 1-120 (2008).

30. Manos, M. M., Leyden, W. A., Murphy, R. C., Terrault, N. A. \& Bell, B. P. Limitations of conventionally derived chronic liver disease mortality rates: results of a comprehensive assessment. Hepatol. Balt. Md. 47, 1150-1157 (2008).

31. Thompson, F. E. et al. Performance of a food-frequency questionnaire in the US NIH-AARP (National Institutes of Health-American Association of Retired Persons) diet and health study. Public Health Nutr. 11, 183-195 (2008).

32. Friday, J. \& Bowman, S. MyPyramid Equivalents Database for USDA Survey Food Codes, 1994-2002 version 1.0. Beltsville, MD: US Department of Agriculture https://www.ars.usda.gov/ARSUserFiles/80400530/pdf/mped/ mped1_doc.pdf (2006).

33. Tippett, K. S. \& Cypel, Y. S. Design and operation: the Continuing Survey of Food Intakes by Individuals and the Diet and Health Knowledge Survey, 199496. US Department of Agriculture, Agricultural Research Service, 1998. 1997;150.

34. Schatzkin, A. et al. Dietary fiber and whole-grain consumption in relation to colorectal cancer in the NIH-AARP diet and health study. Am. J. Clin. Nutr. 85, 1353-1360 (2007).

35. Lipkin, M., Reddy, B., Newmark, H. \& Lamprecht, S. A. Dietary factors in human colorectal cancer. Annu Rev. Nutr. 19, 545-586 (1999).

36. Slavin, J. L. Mechanisms for the impact of whole grain foods on cancer risk. J. Am. Coll. Nutr. 19, 300S-307S (2000).

37. Weickert, M. O. \& Pfeiffer, A. F. H. Impact of dietary fiber consumption on insulin resistance and the prevention of type 2 diabetes. J. Nutr. 148, 7-12 (2018).

38. Willmann, C. et al. Potential effects of reduced red meat compared with increased fiber intake on glucose metabolism and liver fat content: a randomized and controlled dietary intervention study. Am. J. Clin. Nutr. 109, 288-296 (2019).

39. Chen, J.-P., Chen, G.-C., Wang, X.-P., Qin, L. \& Bai, Y. Dietary fiber and metabolic syndrome: a meta-analysis and review of related mechanisms. Nutrients. https://doi.org/10.3390/nu10010024 (2017).

40. Streba, L. A. M., Vere, C. C., Rogoveanu, I. \& Streba, C. T. Nonalcoholic fatty liver disease, metabolic risk factors, and hepatocellular carcinoma: an open question. World J. Gastroenterol. 21, 4103-4110 (2015).

41. Massoud, O. \& Charlton, M. Nonalcoholic fatty liver disease/nonalcoholic steatohepatitis and hepatocellular carcinoma. Clin. Liver Dis. 22, 201-211 (2018).

42. Tan, J. et al. The role of short-chain fatty acids in health and disease. Adv Immunol. 121, 91-119 (2014).

43. Makki, K., Deehan, E. C., Walter, J. \& Bäckhed, F. The impact of dietary fiber on gut microbiota in host health and disease. Cell Host Microbe 23, 705-715 (2018).

44. Desai, M. S. et al. A dietary fiber-deprived gut microbiota degrades the colonic mucus barrier and enhances pathogen susceptibility. Cell 167, 1339-1353.e21 (2016).

45. Kovatcheva-Datchary, P. et al. Dietary fiber-induced improvement in glucose metabolism is associated with increased abundance of Prevotella. Cell Metab. 22, 971-982 (2015).

46. Albillos, A., de Gottardi, A. \& Rescigno, M. The gut-liver axis in liver disease: pathophysiological basis for therapy. J. Hepatol. 72, 558-577 (2020).

47. Dewdney, B., Roberts, A., Qiao, L., George, J. \& Hebbard, L. A sweet connection? fructose's role in hepatocellular carcinoma. Biomolecules. https:// doi.org/10.3390/biom10040496 (2020).

48. Li, W. et al. Effects of hyperglycemia on the progression of tumor diseases. J Exp Clin Cancer Res CR. https://doi.org/10.1186/s13046-019-1309-69 (2019).

49. Ioannou, G. N. Hepatitis B virus in the United States: infection, exposure, and immunity rates in a nationally representative survey. Ann. Intern Med 154, 319-328 (2011).

50. Denniston, M. M. et al. Chronic hepatitis C virus infection in the United States, National Health and Nutrition Examination Survey 2003 to 2010. Ann. Intern Med 160, 293-300 (2014).
51. Campbell, P. T. et al. Body Mass Index, waist circumference, diabetes, and risk of liver cancer for U.S. Adults. Cancer Res 76, 6076-6083 (2016).

52. Petrick, J. L. et al. Tobacco, alcohol use and risk of hepatocellular carcinoma and intrahepatic cholangiocarcinoma: the liver cancer pooling project. $\mathrm{Br}$. J. Cancer 118, 1005-1012 (2018).

53. Petrick, J. L. et al. Coffee consumption and risk of hepatocellular carcinoma and intrahepatic cholangiocarcinoma by sex: the liver cancer pooling project. Cancer Epidemiol. Biomark. Prev. Publ. Am. Assoc. Cancer Res Cosponsored Am. Soc. Prev. Oncol. 24, 1398-1406 (2015).

54. Sui, J. et al. A prospective study of nut consumption and risk of primary hepatocellular carcinoma in the U.S. women and men. Cancer Prev. Res Philos. Pa 12, 367-374 (2019).

55. Yang, W. et al. High dietary intake of vegetable or polyunsaturated fats is associated with reduced risk of hepatocellular carcinoma. Clin Gastroenterol Hepatol. https://doi.org/10.1016/j.cgh.2020.01.003 (2020).

56. Yang, W. et al. A prospective study of dairy product intake and the risk of hepatocellular carcinoma in U.S. men and women. Int J. Cancer 146, 1241-1249 (2020)

57. Ma, Y. et al. Meat intake and risk of hepatocellular carcinoma in two large US prospective cohorts of women and men. Int J Epidemiol. https://doi.org/ 10.1093/ije/dyz146 (2019).

58. Yang, B. et al. Tooth loss and liver cancer incidence in a Finnish cohort. Cancer Causes Control CCC 28, 899-904 (2017).

59. Fedirko, V. et al. Consumption of fish and meats and risk of hepatocellular carcinoma: the European Prospective Investigation into Cancer and Nutrition (EPIC). Ann. Oncol. J. Eur. Soc. Med Oncol. 24, 2166-2173 (2013).

60. Bamia, C. et al. Coffee, tea and decaffeinated coffee in relation to hepatocellular carcinoma in a European population: multi-centre, prospective cohort study. Int J. Cancer 136, 1899-1908 (2015).

\section{Acknowledgements}

This work was supported by the Intramural Research Program of Division of Cancer Epidemiology and Genetics, National Cancer Institute, National Institutes of Health. X.Z. is supported by the National Institutes of Health (grant numbers K07 CA188126, R21CA238651), American Cancer Society Research Scholar Grant (grant number RSG NEC-130476), the Dana-Farber Harvard Cancer Center (DF/HCC) as well as the Zhu Family Center at Harvard Chan School. J.L.P. is supported by the Karin Grunebaum Cancer Research Foundation. Cancer incidence data from the Atlanta metropolitan area were collected by the Georgia Center for Cancer Statistics, Department of Epidemiology, Rollins School of Public Health, Emory University. Cancer incidence data from California were collected by the California Department of Health Services, Cancer Surveillance Section. Cancer incidence data from the Detroit metropolitan area were collected by the Michigan Cancer Surveillance Program, Community Health Administration, State of Michigan. The Florida cancer incidence data used in this report were collected by the Florida Cancer Data System (FCDS) under contract with the Florida Department of Health (FDOH). Cancer incidence data from Louisiana were collected by the Louisiana Tumor Registry, Louisiana State University Medical Center in New Orleans. Cancer incidence data from the New Jersey were collected by New Jersey State Cancer Registry, Cancer Epidemiology Services, New Jersey State Department of Health and Senior Services. Cancer incidence data from North Carolina were collected by the North Carolina Central Cancer Registry. Cancer incidence data from Pennsylvania were supplied by the Division of Health Statistics and Research, Pennsylvania Department of Health, Harrisburg, Pennsylvania. The Pennsylvania Department of Health specifically disclaims responsibility for any analyses, interpretations, or conclusions. Cancer incidence data from the Arizona were collected by Arizona Cancer Registry, Division of Public Health Services, Arizona Department of Health Services. Cancer incidence data from Texas were collected by the Texas Cancer Registry, Cancer Epidemiology and Surveillance Branch, Texas Department of State Health Services. Cancer incidence data from Nevada were collected by the Nevada Central Cancer Registry, Center for Health Data and Research, Bureau of Health Planning and Statistics, State Health Division, State of Nevada Department of Health and Human Services. (The above information can also be found at: https://dietandhealth.cancer.gov/acknowledgement.html).

\section{Author contributions}

X.Z. designed the study. J.L.P., L.M.L. and K.A.M. were involved in the data collection. X.L., W.Y., J.L.P., E.G. and X.Z. provided statistical expertise. X.L. wrote the first draft of the manuscript. W.Y., J.L.P., L.M.L., K.A.M., P.T.C., Z.F.Z., W.W., N.H., E.G. and X.Z. revised the manuscript or provided comments or suggestions. All authors contributed to the interpretation of the results and critical revision of the manuscript for important intellectual content and approved the final version of the manuscript.

\section{Competing interests}

The authors declare no competing interests. 


\section{Additional information}

Supplementary information The online version contains supplementary material available at https://doi.org/10.1038/s41467-021-26448-9.

Correspondence and requests for materials should be addressed to Xuehong Zhang

Peer review information Nature Communications thanks Elena George and the other,anonymous, reviewer(s) for their contribution to the peer review of this work

Reprints and permission information is available at http://www.nature.com/reprints

Publisher's note Springer Nature remains neutral with regard to jurisdictional claims in published maps and institutional affiliations. (c) (1) Open Access This article is licensed under a Creative Commons Attribution 4.0 International License, which permits use, sharing, adaptation, distribution and reproduction in any medium or format, as long as you give appropriate credit to the original author(s) and the source, provide a link to the Creative Commons license, and indicate if changes were made. The images or other third party material in this article are included in the article's Creative Commons license, unless indicated otherwise in a credit line to the material. If material is not included in the article's Creative Commons license and your intended use is not permitted by statutory regulation or exceeds the permitted use, you will need to obtain permission directly from the copyright holder. To view a copy of this license, visit http://creativecommons.org/ licenses/by/4.0/.

(c) The Author(s) 2021 